\title{
Prevalência de lesões na coluna de docentes da rede de ensino público da cidade de Montes Claros - MG
}

\author{
Prevalence of injuries in the column of teachers of the network of public education of the city \\ of Montes Claros - MG
}
Prevalencia de lesiones en la columna de docentes de la red de ensino público de la ciudad de Montes Claros - MG

João Jefferson Carneiro Corrêa ${ }^{1 *}$, João Victor Cardozo Silva ${ }^{1}$, Débora Ribeiro Vieira ${ }^{2}$, Júnia Caldeira Guimarães ${ }^{3}$, Eric Hudson Evangelista e Souza ${ }^{1}$, Saulo Daniel Mendes Cunha'.

\begin{abstract}
RESUMO
Objetivos: Determinar a prevalência de lesões na coluna de docentes da rede pública de ensino da cidade de Montes Claros- MG. Métodos: Trata-se de um estudo quantitativo descritivo com uma população de 256 professores do ensino médio e fundamental, das 4 maiores escolas municipais da cidade. O questionário aplicado foi o Nordic Musculo skeletal Questionnaire, que avalia e mensura o nível de dores musculoesqueléticas. Foi Utilizado se o aplicativo Microsoft Exceß 2016 para confecção da planilha dos dados coletados, e das funções médias e desvio padrão para obter os resultados. Resultados: Em posse dos dados, averiguou que $58,33 \%$ dos professores apresentaram dor na coluna, sendo a região torácica mais acometida, Porém, poucos docentes procuraram um profissional da saúde por este motivo. Conclusões: Conforme a amostra pesquisada, a prevalência de dores na coluna em professores é alta, sendo assim deve por parte dos professores e do setor público estadual, buscar meios que venham a reverter este quadro.
\end{abstract}

Palavras Chave: Professores, Coluna, Lesões.

\begin{abstract}
Objectives: To determine the prevalence of injuries in the teaching staff of the public school system in the city of Montes Claros, MG. Methods: This is a quantitative descriptive study with a population of 256 high school and elementary teachers from the 4 largest municipal schools in the city. The questionnaire applied was the Nordic Musculo skeletal Questionnaire, which evaluates and measures the level of musculoskeletal pain. The Microsoft Excel® 2016 application was used to compile the spreadsheet of the data collected, and the mean and standard deviation functions to obtain the results. Results: In the possession of the data, $58.33 \%$ of the teachers presented pain in the spine, and the thoracic region was more affected. However, few teachers sought a health professional for this reason. Conclusions: According to the sample surveyed, the prevalence of pain in the spine in teachers is high, so it should be sought by teachers and the state public sector to find means that will reverse this situation.
\end{abstract}

Key words: Teachers, Column, Injuries.

\section{RESUMEN}

Objetivos: Determinar la prevalencia de lesiones en la columna de docentes de la red pública de enseñanza de la ciudad de Montes Claros-MG. Métodos: Se trata de un estudio cuantitativo descriptivo con una población de 256 profesores de la enseñanza media y fundamental, de las 4 mayores escuelas municipales de la ciudad. El

\footnotetext{
${ }^{1}$ Universidade Estadual de Montes Claros (UNIMONTES), Montes Claros-MG. *E-mail:joaojefferson5@hotmail.com

${ }^{2}$ Faculdades Integradas Pitágoras de Montes Claros- MG

3Instituto Israelita de Ensino e Pesquisa Albert Einstein
}

SUBMETIDO EM: 5/2019

ACEITO EM: 6/2019

PUBLICADO EM: 8/2019

REAS/EJCH | Vol.11(13) | e926 | DOI: https://doi.org/10.25248/reas.e926.2019 Página 1 de 8 
cuestionario aplicado fue el Nordic Musculo skeletal Questionnaire, que evalúa y mensura el nivel de dolores musculo esqueléticos. Se utilizó si la aplicación Microsoft Excel® 2016 para la confección de la hoja de cálculo de los datos recopilados, y las funciones medias y la desviación estándar para obtener los resultados. Resultados: En posesión de los datos, averiguó que 58,33\% de los profesores presentaron dolor en la columna, siendo la región torácica más acometida, pero pocos docentes buscaban a un profesional de la salud por este motivo. Conclusiones: Conforme a la muestra investigada, la prevalencia de dolores en la columna en profesores es alta, siendo así por parte de los profesores y del sector público estadual, buscar medios que vayan a revertir este cuadro.

Palabras clave: Profesores, Columna, Lesiones.

\section{INTRODUÇÃO}

Uma das características marcantes das atividades docentes é "a mobilização física e intelectual para o exercício da docência na escola, no domicílio ou em lugares determinados para preparação de aulas, correções, estudos, reuniões, etc" (SOUZA A, 2008). Este conceito só expõe ainda mais a posição primordial do docente na sociedade e quanto a sua formação. Porém, por diversos fatores como falta de investimento e criação de novas políticas públicas, que protegem esta classe, eles acabam sendo desvalorizados. Como resultado, ocorre a sobrecarga de trabalho dos educadores e isto ecoa na sua saúde, (SANTOS M e MARQUES A, 2013) ensinar é, em geral, uma atividade altamente estressante, com repercussões evidentes na saúde física, mental e no próprio desempenho desses profissional, que além de ministrar aulas, realizam trabalhos administrativos, planejamentos, orientam os alunos e interagem com seus pais.

Barros M, et al. (2007), Desgastes osteomusculares e transtornos mentais, como apatia, estresse, desesperança e desânimo, são formas de adoecimento que têm sido identificadas em professores. Estas patologias podem estar agregadas às condições desfavoráveis exercidas pelos docentes como número de alunos abundantes, longa jornadas de trabalho, baixos salários, ficam muito tempo em pé, assentos nada ergonômicos entre outros.

De acordo com Branco JC, et al. (2011), no seu estudo com professores do ensino fundamental, mencionou que as dores na região tóraco-lombar pode estar relacionado a inúmeros fatores da rotina do docente, frisando o fato de trabalhar muitas horas com o membro superior elevado associado à rotação de tronco com o pescoço levemente inclinado, resultando à musculatura cervical, escapular e tóraco- lombar o desenvolvimento de sintomas dolorosos.

Neste cenário de múltiplas tarefas solicitado pela profissão, com o passar do tempo, sem tomar as devidas precauções, pode acarretar em dores, lesões, e pode converter em uma Doença Osteomuscular relacionado ao trabalho (DORT). Uma doença de cunho ocupacional, definida pelo Ministério da Saúde, como aqueles danos decorrentes da utilização excessiva do sistema músculo esquelética e da falta de tempo para recuperação. Caracterizam-se pela ocorrência de vários sintomas, de aparecimento quase sempre em estágio avançado, que ocorrem geralmente nos membros superiores, tais como dor, sensação de peso e fadiga. São as doenças que mais afetam os brasileiros, e que entre o período de 2007 a 2016 houve o aumento de 3.212 para 9.122 casos. Quase triplicando os casos, (MS, 2018).

A DORT pode acometer várias partes do músculo esquelético, e uma delas sendo a coluna vertebral, ou seja, patologias como cervicalgia e lombalgia. A prevalência de cervicalgia, dor na cervical, no âmbito da medicina ocupacional vem aumentando significativamente, sendo considerada como um dos grandes problemas da sociedade moderna (BRING G e BRING J, 1995).

Há uma grande dificuldade em obter dados fiéis para a estimativa da real prevalência das cervicalgias, visto que se trata de um grupo de doenças com aspectos clínicos multifatoriais, envolvendo desde fatores de risco individuais, como características físicas e psicossociais, até fatores relacionados com a ergonomia e atividades laborativas (HALES TR e BERNARD BP, 1996). 
Já a lombalgia, é queixa mais freqüente de dores na coluna vertebral, sendo que $65 \%$ a $90 \%$ dos adultos poderão sofrer um episódio de lombalgia ao longo da vida, com incidência entre $40 \%$ e $80 \%$ em várias populações estudadas. A dor lombar crônica pode ser causada por doenças inflamatórias, degenerativas, neoplásicas, defeitos congênitos, debilidade muscular, predisposição reumática, sinais de degeneração da coluna ou dos discos intervertebrais e outras (NATOUR J, et al., 2004).

A total sintonia que ocorre entre as más condições oferecidas para o exercício da profissão de docente, que envolve tanto sobrecarga de trabalho e sua realização ergonômica de forma errônea, e as lesões que podem ocorrer diante desse descaso, muitas das vezes impacta de tal maneira que leva ao resultado mais prejudicial, não apenas ao professor, mas para o ensino e formação da sociedade como um todo. As condições de trabalho constituem um dos fatores principais do mal-estar docente. Tais condições afetam a saúde física e mental dos professores levando-os ao absenteísmo e, às vezes, ao abandono da profissão. (ESTEVE J, 1999).

Por fim, os trabalhadores têm que se adaptar às tecnologias e se atualizar perante um mercado competitivo. Diante destas situações, o ser humano está envolvido num processo complexo e dinâmico que abrange as condições somáticas, os processos cognitivos e emocionais, e as questões sociais, (CHIAVEGATO F, et al., 2004).

A presença de dores e limitações, decorrentes da doença, contribui para o surgimento de sintomas depressivos e de ansiedade, acompanhada de angústia e medo em relação a um futuro incerto. A partir destas modificações, o trabalhador perde um pouco da sua identidade e ganha insegurança no ambiente de trabalho, familiar e social, (BURTON J, et al., 2002).

Mediante este contexto, esse trabalho tem por objetivo determinar a prevalência de lesões na coluna de docentes da rede de ensino público da cidade de Montes Claros - MG.

\section{METODOLOGIA}

Trata-se de um estudo quantitativo, com organização da amostra por conveniência, com base no número de docentes atuantes nas instituições escolares. A população desta pesquisa foi composta por 256 professores atuantes na rede pública da cidade de Montes Claros - MG, dados que foram obtidos através da Superintendência Regional de Ensino da cidade após toda a documentação necessária ser apresentado.

As 4 instituições públicas da cidade de Montes Claros com maior número de docentes foram indicadas para que os dados fossem coletados.

O cálculo amostral com índice de confiança de 95\% e erro amostral de 5\% revelou um número de 256 docentes a serem pesquisados.

Inicialmente nas escolas e aos professores, ocorreu o esclarecimento do tema e da intenção da pesquisa, logo após recolhemos as assinaturas de ambos, de concordância da instituição e o Termo de concordância livre e esclarecida (TCLE) dos professores.

A aplicação do questionário Nordic Musculo skeletal Questionnaire traduzido, validado no Brasil por Pinheiro, Tróccoli e Carvalho (2002), e dos dados pessoais dos professores, foram em uma segunda visita por parte dos pesquisadores e na data combinada anteriormente com todos da instituição. Este questionário nórdico é um instrumento que consiste em um conjunto de escolhas múltiplas ou binárias referentes à ocorrência de sintomas nas diversas regiões anatômicas nas quais são mais comuns, nele os selecionados, no caso do estudo os docentes, relatam a ocorrência dos sintomas considerando os 12 meses e os sete dias precedentes à entrevista, bem como informaram a ocorrência de afastamento das atividades rotineiras no último ano.

Os critérios de inclusão adquiridos foram Ser professores do ensino público de ensino, que tenham mais de cinco anos de atuação na área, que não apresentaram diagnóstico de doenças ocupacionais crônicas, não apresentaram atestado médico por motivo de problemas relacionados ao trabalho nos últimos 6 meses a data de aplicação do questionário, não estavam em tratamento de lesões/doenças ocupacionais, e os que demonstraram interesse e voluntariedade em participar da pesquisa. Além de ter formação acadêmica em 
qualquer área de ensino e aceitar assinar o termo de consentimento livre e esclarecido. E critérios de exclusão da pesquisa foram professores com licença escolar, professores que se recusaram em participar da pesquisa; os e professores que não tiverem no mínimo cinco anos de atuação na área de docência e o que não apresentaram o Termo de concordância livre e esclarecido (TCLE) assinado.

O número total de docentes que foram aptos a atuar na pesquisa respondendo ao questionário foi de 172 (67.18\%). Durante as visitas de coleta houve por parte de 84 (32.81\%) professores a desistência de participar, cada um deles alegando motivos diversos e pessoais, sendo respeitado pelos pesquisadores, já que é uma possibilidade de acordo com os critérios de exclusão.

O projeto foi aprovado pelo Comitê de Ética em Pesquisa com Seres Humanos, e os dados foram coletados após consentimento informado dos professores, preservando o anonimato. A confidencialidade das informações, sendo tão somente utilizados para fins científicos e o direito de recusa em participar foram plenamente garantidos. A aprovação do projeto foi confirmada no dia 07/02/2019, recebendo o número do parecer 3.037.472.

Após o término da coleta de dados realizada, as informações foram transcritas para um banco de dados na planilha eletrônica do Microsoft Exce/® (Versão PT/2016), posteriormente utilizando das funções de média e desvio padrão houve a análise estatística e obteve-se os resultados almejados.

\section{RESULTADOS E DISCUSSÃo}

Entre os docentes investigados 119 (69.18\%) eram do sexo feminino e 53 (30.81\%) do sexo masculino (Tabela 1). Neste sentido, os resultados obtidos na pesquisa de Souza A e Gouveia A (2011), relatam com base nos questionários de contexto do Sistema de Avaliação da Educação Básica (SAEB), a atuação das mulheres no magistério cresceu de 71\% para 74\% entre 1997 e 2007; Gatti B e Barreto E (2009), utilizando a Pesquisa Nacional por Amostragem Doméstica (PNAD) de 2006, expõem a presença feminina na profissão docente na educação básica em $67 \%$.

Tabela 1 - Descrição da Amostra.

\begin{tabular}{lccc}
\multicolumn{1}{c}{ Variáveis } & N & Média & Desvio Padrão \\
\hline $\begin{array}{l}\text { Sexo } \\
\text { Masculino }\end{array}$ & 53 & & \\
$\begin{array}{l}\text { Feminino } \\
\text { Idade }\end{array}$ & 119 & & \\
\hline $\begin{array}{l}\text { ldade Máx. } \\
\text { Idade Mín. } \\
\text { Carga Horária }\end{array}$ & 62 anos & 42,20 anos & $\pm 8,78$ \\
\hline $\begin{array}{l}\text { Tempo de Docência } \\
\text { Instituições trabalhadas }\end{array}$ & 25 anos & $24,14 \mathrm{hs} /$ semanais & \\
\hline
\end{tabular}

Fonte: Superintendência Regional de Ensino da cidade de Montes Claros - MG, 2018.

A média de idade dos participantes desta pesquisa foi de 42,20 anos $( \pm 8,78)$. De acordo com INEP/MEC, (2016), ocorre uma concentração de docentes nas faixas etárias de 26 a 35 anos e de 36 a 45 anos (29,7\% e $34,1 \%$ do total, respectivamente.

O tempo médio na função de professor nesta pesquisa foi de 15,65 anos $( \pm 7,30)$. Sendo o maior tempo de docência 36 anos e o menor de 05 anos. Os resultados encontrados por Souza A e Gouveia A (2011), no estudo sobre políticas educacionais, relatam um aumento da experiência profissional por parte dos professores, uma vez que em 1997 eram 14,6\% dos docentes com mais de 21 anos de trabalho docente, enquanto em 2007, este grupo representava mais de $1 / 4$ do total da população. E, na outra ponta, enquanto em 1997, 27,6\% dos docentes tinham menos de 5 anos de atuação profissional, em 2007, este grupo cai para 17,1\%.

A média da carga horária semanal dos professores entrevistados foi de 24,14 horas semanais $( \pm 10,90)$. Um resultado semelhante no estudo da Organisation for Economic Co-operation and Development (OECD), 
com resultados analisados de coletas de 14 mil docentes, chegou ao quociente de 25 horas semanais, sendo 6 horas a mais do que outros países que participaram. Já no estudo de Faria e Rachid (2010), sobre análise de contratos de trabalho no ensino público, encontrou uma maior concentração deles na faixa de mais de 44 horas semanais, isto é, 32 dos 95 docentes se encontram nessa faixa, representando 33,7\%.

A maioria dos professores que responderam o questionário trabalha em 1 instituição de ensino. Neste sentido, ao contrário do resultado da pesquisa Talis (2013), realizada pela $O E C D$, e coordenada aqui no Brasil pelo Instituto Nacional de Estudos e Pesquisas Educacionais (INEP), que relata que apenas $40 \%$ dos professores brasileiros atuantes no ensino têm dedicação a somente uma instituição, contra $82 \%$ das outras nações que estão no estudo.

Gráfico 1 - Prevalência de dor, formigamento e dormência na região da coluna nos últimos 12 meses.

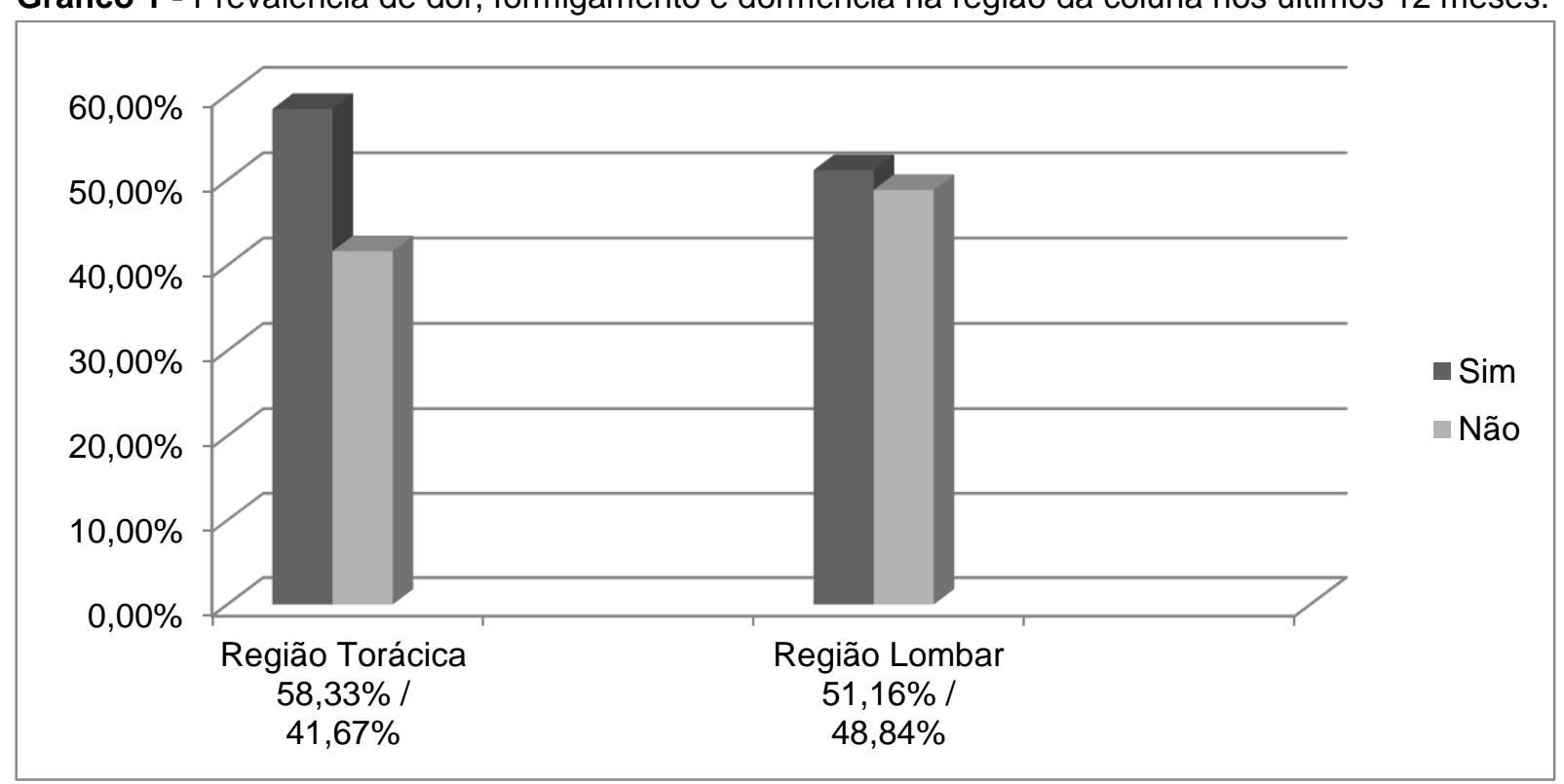

Fonte: Dados da pesquisa, 2019.

Do total de docentes investigados, no setor da coluna, o presente estudo apresentou que 100 (58,33\%) professores responderam SIM, e $72(41,67 \%)$ responderam NÃO em relação a região torácica, já na região lombar, 84 pessoas $(51,16 \%)$ responderam SIM e 88 pessoas $(48,84 \%)$ respondendo NÃO para prevalência de dor, formigamento e dormência nos últimos 12 meses (Gráfico 1).

Para complementar, Maehler P (2003) explica que os relatos de dor no dorso e região lombar são comuns quando se utiliza, por longos períodos, a postura ortostática, pois nessa postura ocorre mais atividade nos eretores da espinha, o que gera fadiga muscular e consequente dor; outra consequência à fadiga dos músculos posturais é a má postura que sobrecarrega ligamentos e cápsulas e aumenta o estresse sobre os discos vertebrais.

Dessas 172 pessoas, apenas 31 (18,02\%) responderam SIM e 141 (82,45\%) para a segunda questão do questionário nórdico, que se refere ao impedimento para realizar trabalhos normais como, por exemplo: trabalhar, realizar atividades domésticas e de lazer por causa deste problema relacionados à coluna (Gráfico 2). Segundo o Ministério do Trabalho (2017) no Brasil a dorsalgia é o principal causa de afastamento do trabalho, por volta de 116 mil pessoas foram afastadas por 15 dias do trabalho, porém a classe de docentes não é o ofício que está nas primeiras colocações dos cargos mais acionados por afastamento.

Complementando sobre o assunto Abyholm AS e Hjortahl P (1999), diz em seu estudo que este tipo de complicação acomete principalmente pessoas que estão em fase economicamente ativa e que é altamente incapacitante sendo uma das principais causas de afastamento. A dor sofrida de forma crônica pelo indivíduo pode acarretar distúrbios, depressão e vários outros problemas que afetam sua vida. 
Fernandes $\mathrm{H}$, et al. (2009) em seu estudo com professores de Natal- RN, 47,7 \% relataram que a presença da sintomatologia osteomuscular nos últimos 12 meses impediu a realização de atividades da vida diária.

Gráfico 2 - Impedimento de realizar atividades normais por causa desse problema na coluna.

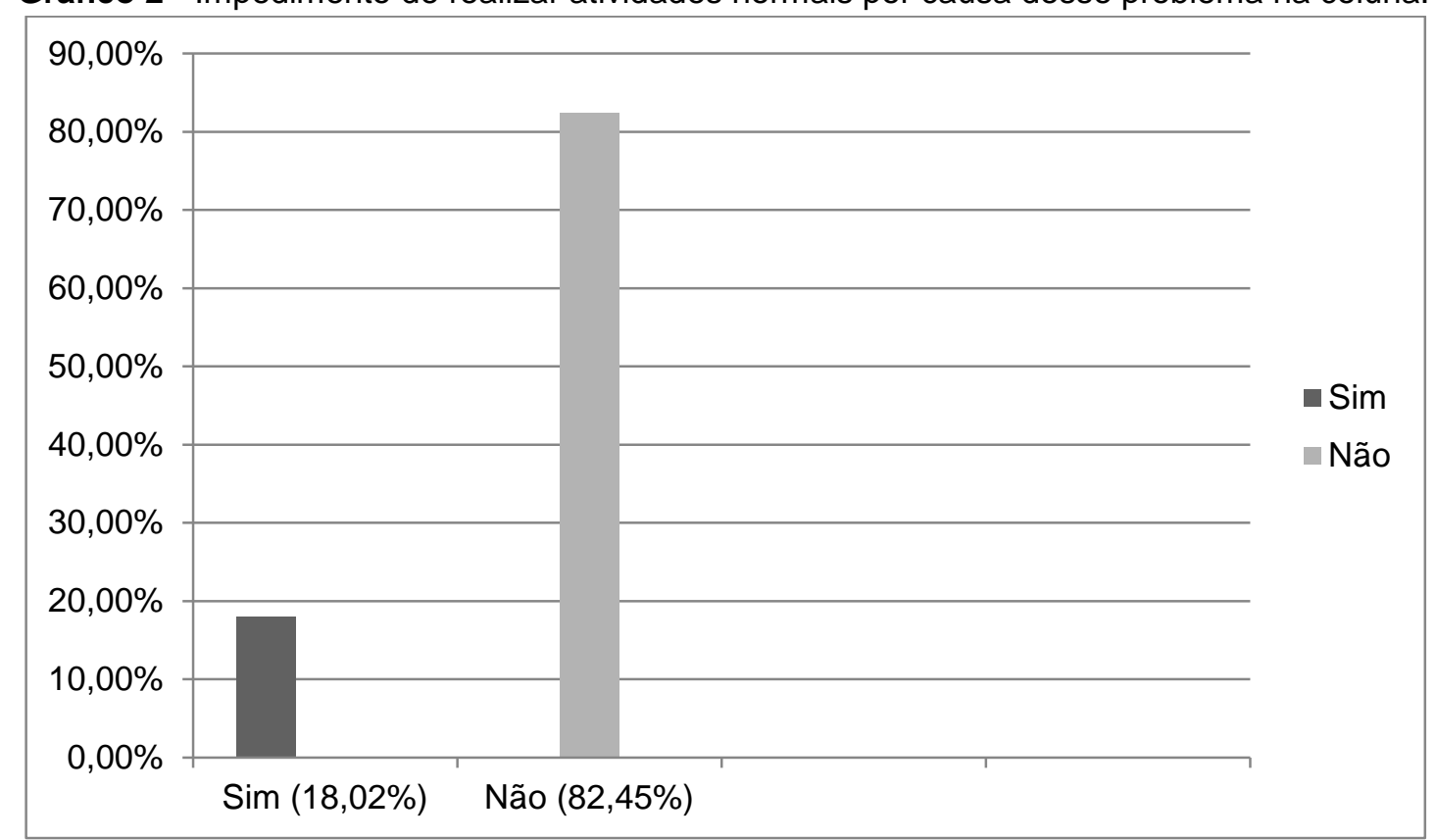

Fonte: Dados da pesquisa, 2019.

Apesar do grande número de docentes com sintomas de dores osteomuscular na coluna vertebral neste estudo, a grande maioria cerca de $150(84,30 \%)$ NÃO procurou ajuda de um profissional capacitado da saúde, nos últimos 12 meses, para identificar o real motivo das dores, contra 27 (15,7\%) que responderam SIM (Gráfico 3).

Gráfico 3 - Consultou algum profissional da saúde por causa dessa condição.

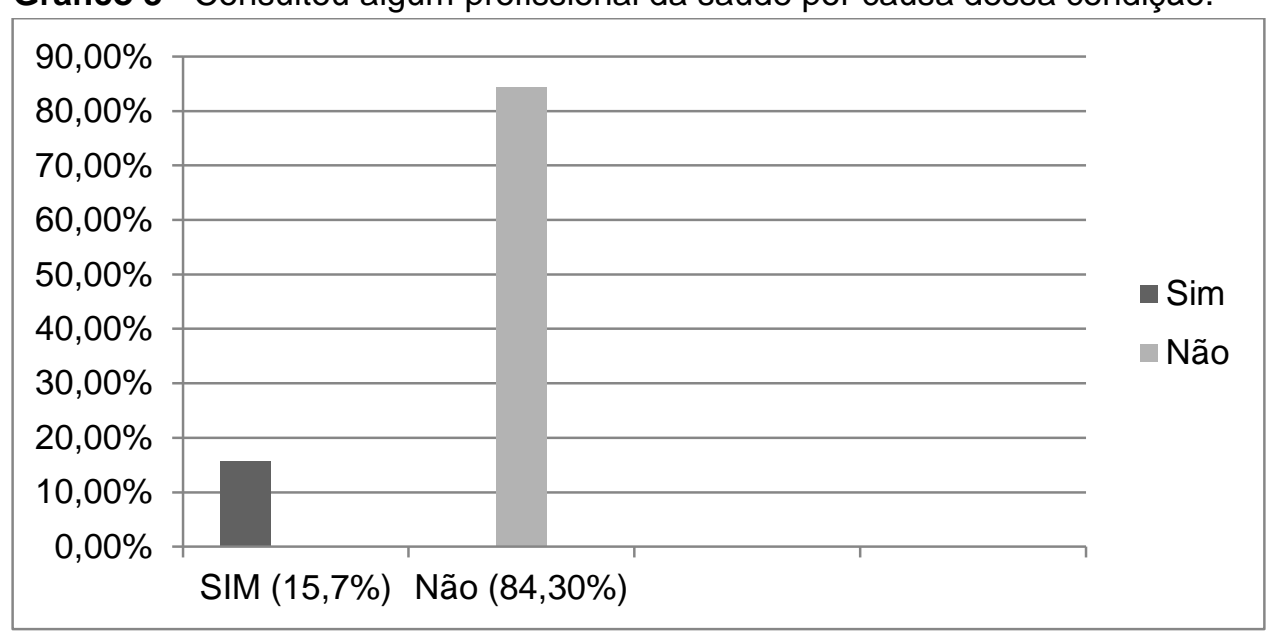

Fonte: Dados da pesquisa, 2019.

Estudo realizado pelo Instituto Data Popular vai de encontro ao resultado já que encontrou que apenas 6 em cada 10 brasileiros só consultam com um profissional da saúde quando estão doentes. Evidenciando que 0 brasileiro não tem o costume de fazer exames regulares de prevenção, o que pode ocasionar em problemas maiores e irreversíveis no futuro. 
Em um estudo do Melo, et al. (2010), realizado com professores do Ensino Fundamental das Escolas da rede Municipal da cidade de Catalão (GO), do total de investigados, $96 \%$ dos participantes relataram ter realizado consulta a algum profissional da saúde devido ao referido problema.

Analisando o último tópico do Questionário Nórdico, 29 (17\%) dos entrevistados afirmaram que tiveram algum problema na região da coluna nos últimos 7 dias, contra $143(83 \%)$ que negaram. Esse resultado retirado dos dados coletados sugere que apesar da alta prevalência de dor na coluna, poucos sentem as dores de forma crônica (Gráfico 4).

Gráfico 4 - Nos últimos 7 dias, você teve algum problema na coluna?

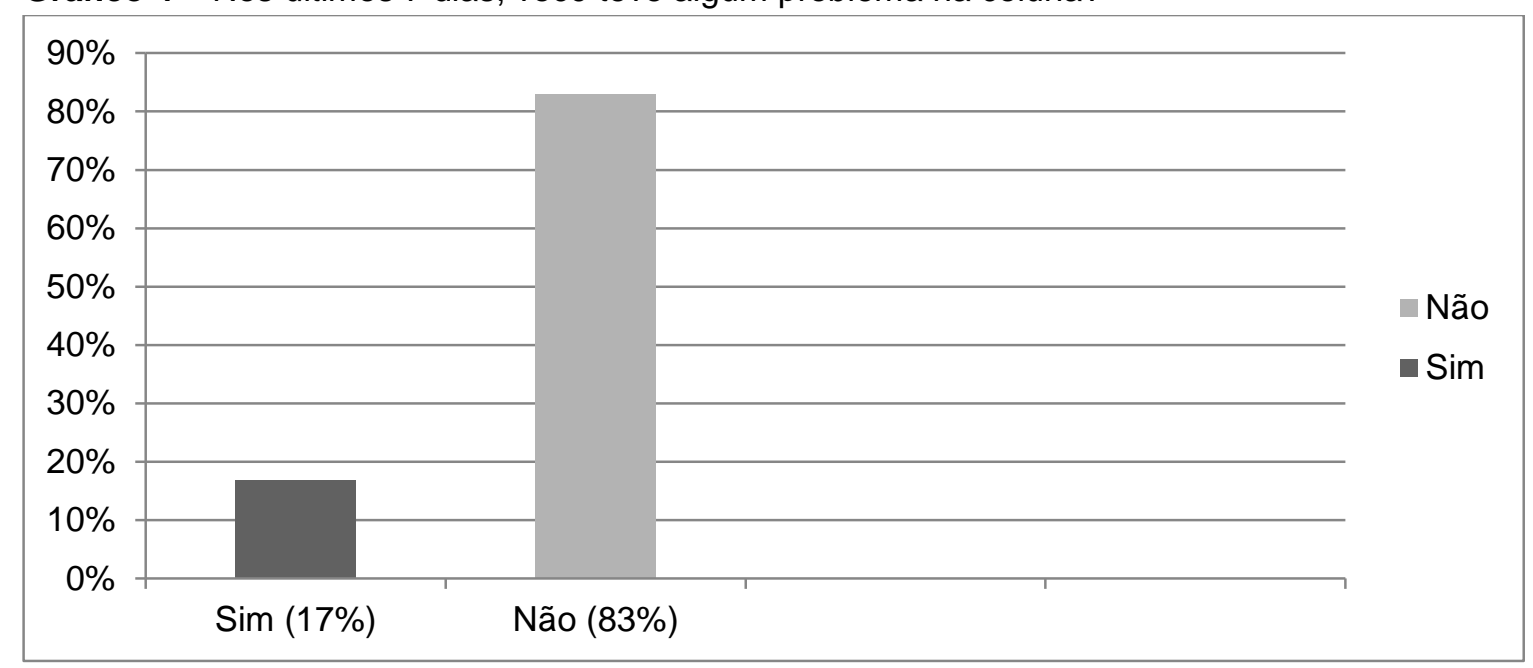

Fonte: Dados da pesquisa, 2019.

Segundo dados da Pesquisa Nacional de Saúde (2014), revelam que cerca de 57,4 milhões de brasileiros têm pelo menos uma doença crônica, sendo 33,4 milhões de mulheres e 23 milhões homens. Resultados estes associados ao estilo de vida como excesso de peso, sedentarismo dentre outros.

A título de comparação, o estudo realizado por Carvalho A e Alexandre N., (2006), que também aplicou o questionário Nordic Musculo skeletal para professores do ensino fundamental, obteve no seu desfecho a confirmação dolorosa de $27,4 \%$ na mesma região do corpo.

\section{CONCLUSÃO}

Mediante os dados expostos, o presente estudo apontou uma alta prevalência de lesões na coluna vertebral de professores de escolas públicas da cidade de Montes Claros - MG. Com base neste resultado que identifica pontualmente o problema de saúde na coluna sofrida pelos docentes das escolas estaduais, percebemos a importância da atuação do setor da gestão publica do estado de Minas Gerais. Desta forma, é necessário planejar e implantar uma nova política de valorização da Carreira docente, que venha a melhorar as condições de trabalho. Outros pontos a serem destacados, dizem respeito à implantação de programa de aprendizado de postura humana no ambiente de trabalho e mudanças de hábitos no seu dia a dia.

\section{REFERÊNCIAS}

1. ABYHOLM AS, HJORTAHL P. The pain takes hold of life: a qualitative study of how patients with chro nic back pain experience and cope with their life situation. Tidsskr Nor Laegeforen 1999; 30 : 1624 - 9

2. BARROS M, et al. Saúde e trabalho docente: a escola como produtora de novas formas de vida. Trabalho, Educação e Saúde, 2016; 5(1), 103-123.

3. BRANCO JC, et al. Prevalência de sintomas osteomusculares em professores de escolas públicas e privadas do ensino fundamental. Fisioter Mov. 2011; 24(2):307-14. 
4. BRING G, BRING J. Neck pain in general population. Spine, 1995. 20:624-7.

5. BURTON J, et al. Evaluating the social and economic consequences of workplace injury and illness. New Zeland : workplace Safety and health. 2002.

6. CARVALHO A. E ALEXANDRE N., Sintomas osteomusculares em professores do ensino fundamental. Revista Brasileira de Fisioterapia, 2006; São Carlos, v.10, n.1.

7. CHIAVEGATO et al. LER/DORT: multifatorialidade etiológica e modelos explicativos, Interface - Comunic., Saúde, Educ., set.2003-fev.2004; 8(14):149-62.

8. ESTEVE J. Mal-estar docente: a sala de aula e a saúde dos professores. 1999. São Paulo: Edusc.

9. EXAME. 2019. In: O que precisa ser feito para que brasileiros visitem médicos periodicamente?. Disponível em : http://www.brasil.gov.br/economia-e-emprego/2017/04/dor-nas-costas-e-a-maior-causa-de-afastamento-dotrabalho. Acesso em: 20 julho 2019.

10. FARIA G, RACHID A. Análise da diversidade de contratos de trabalho no ensino público. Revista da ABET, 2010; São Paulo, v. 9, n. 1, p. 148-164.

11. FERNANDES H. et al. Fatores Associados à Prevalência de Sintomas Osteomusculares em Professores. Rev. salud pública, 2009; 11 (2): 256-267.

12. GATTI B, BARRETO E. S. Professores do Brasil: impasses e desafios. Brasília: UNESCO, 2009.

13. GOVERNO DO BRASIL. 2018. In: Dor nas costas é a maior causa de afastamento do trabalho. Disponível em : http://www.brasil.gov.br/economia-e-emprego/2017/04/dor-nas-costas-e-a-maior-causa-de-afastamento-dotrabalho. Acesso em: 13 maio 2019.

14. HALES TR, BERNARD BP. Epidemiology of work-related musculoskeletal disorders. Orthop Clin North Am 1996; 27 : 679-709.

15. INEP/MEC, Censo escolar da educação básica 2016 Notas Estatísticas. Brasília-DF, 2017.

16. MAEHLER P. Estudo das sobrecargas posturais em acadêmicos de odontologia da Universidade Estadual do Oeste do Paraná-Unioste Cascavel [Monografia], 2003; Cascavel: Curso de Odontologia, Universidade Estadual do Oeste do Paraná;

17. MELO et al. Prevalência de lesões osteomusculares em professores do ensino fundamental. Revista Eletrônica "Saúde CESUC", 2010; № 01.

18. MINISTÉRIO DA SAÚDE. 2018. In: LER e DORT são as doenças que mais acometem os trabalhadores. Disponível em : http://portalms.saude.gov.br/noticias/agencia-saude/45404-ler-e-dort-sao-as-doencas-que-mais-acometem-ostrabalhadores-aponta-estudo. Acesso em: 09 maio 2019.

19. MINISTÉRIO DA SAÚDE. 2014. In: 57,4 milhões de brasileiros têm pelo menos uma doença crônica. Disponível em : http://www.blog.saude.gov.br/index.php/34861-57-4-milhoes-de-brasileiros-tem-pelo-menos-uma-doenca-cronica. Acesso em: 20 junho 2019.

20. NATOUR J, et al. Coluna Vertebral, Conhecimentos básicos, etceteras Editora de Livros e Revistas, 2004.

21. OECD, Teaching and Learning International Survey TALIS 2013, 2013; Paris.

22. SOUZA A. Condições de trabalho na carreira docente: comparação Brasil-França. In: COSTA, A. et al. Mercado de trabalho e gênero: comparações internacionais. Rio de Janeiro: Fundação Getúlio Vargas, 2008. p. 355-370.

23. SOUZA A, GOUVEIA A. Os trabalhadores docentes da educação básica no Brasil em uma leitura possível das políticas educacionais. Arquivos Analíticos de Políticas Educativas. Revista acadêmica, 2011. v. 19, n. 35, dez.

24. SANTOS M, MARQUES A. Condições de saúde, estilo de vida e características de trabalho de professores de uma cidade do sul do Brasil. Ciência \& Saúde Coletiva, 2013; v. 18, p. 837-846.

25. World Health Organization. Identification and control of work - related diseases. Geneva: World Health Organization; 1985; (Technical Report Series 714). 